\title{
Invariant Differential Operators in Noncommutative Quantum Group Setting
}

\author{
Vladimir Dobrev* \\ Bulgarian Academy of Sciences, Institute for Nuclear Research and Nuclear Energy \\ E-mail: vkdobrevdyahoo.com
}

In the present talk we review the progress of the project of construction of invariant differential operators for non-compact semisimple Lie groups and their quantum group deformations. The main examples are the conformal quantum group and the q-Maxwell equations hierarchy.

Proceedings of the Corfu Summer Institute 2015 "School and Workshops on Elementary Particle Physics and Gravity"

1-27 September 2015

Corfu, Greece

${ }^{*}$ Speaker. 


\section{Introduction}

1.1. Invariant differential operators play very important role in the description of physical symmetries - starting from the early occurrences in the Maxwell, d'Allembert, Dirac, equations, to the latest applications of (super-)differential and difference operators in conformal field theory, supergravity and string theory and their deformations. Thus, it is important for the applications in physics to study systematically such operators and, in particular, their deformations in the setting of quantum groups.

The approach to this problem used here relies on the following classical considerations. Consider a semisimple Lie group $G$ and two representations $T, T^{\prime}$ acting in the representation spaces $C, C^{\prime}$, which may be Hilbert, Fréchet, etc. An intertwining operator $\mathscr{I}$ for these two representations is a continuous linear map so that:

$$
\mathscr{I}: C \longrightarrow C^{\prime}, \quad \mathscr{I} \circ T(g)=T^{\prime}(g) \circ \mathscr{I}, \quad \forall g \in G
$$

Such equations exist also for more general symmetries. However, if $G$ is semisimple (even reductive) then there exists canonical ways for the construction of all intertwining operators and thus, of the $G$ - invariant equations. These operators are of two types - differential and integral. Here we are interested in the invariant differential operators. Due to the lack of space we refer to [W] for more motivations and recent literature.

1.2. We shall apply a procedure [[] which is rather algebraic and can be generalized almost straightforwardly to quantum groups. We sketch this procedure illustrating the general notions with the conformal group $S U(2,2)$.

Let $G$ be a real semisimple Lie group. Let $\mathscr{G}$ be the Lie algebra of $G$. We shall use the so-called Bruhat decompositions of $\mathscr{G}$

$$
\mathscr{G}=\mathscr{N}^{+} \oplus \mathscr{M} \oplus \mathscr{A} \oplus \mathscr{N}^{-}
$$

(considered as direct sum of linear spaces), where $\mathscr{A}$ is a noncompact abelian subalgebra, $\mathscr{M}$ (a reductive Lie algebra) is the centralizer of $\mathscr{A}$ in $\mathscr{G}(\bmod \mathscr{A})$, and $\mathscr{N}^{+}, \mathscr{N}^{-}$, resp., are nilpotent subalgebras forming the positive, negative, resp., root spaces of the restricted root system $(\mathscr{G}, \mathscr{A})$. For the conformal group the subalgebras $\mathscr{N}^{-}, \mathscr{M}, \mathscr{A}, \mathscr{N}^{+}$may be chosen to be the subalgebras of translations, Lorentz transformations, dilatations, special conformal transformations, resp. Note that $\tilde{\mathscr{P}}^{ \pm}=\mathscr{N}^{ \pm} \oplus \mathscr{M} \oplus \mathscr{A}$ are subalgebras of $\mathscr{G}$, the so-called parabolic subalgebras. With the above identification $\tilde{\mathscr{P}}^{ \pm}$are maximal conjugate parabolic subalgebras; otherwise $\tilde{\mathscr{P}}^{+}$is called the Weyl algebra (comprising the Poincaré algebra and the dilatations).

Let us now introduce the corresponding subgroups of $G$. Let $K$ denote the maximal compact subgroup of $G$, and let $\mathscr{K}$ denote the Lie algebra of $K$. Then we have the simply connected subgroups $A=\exp (\mathscr{A}), N^{ \pm}=\exp \left(\mathscr{N}^{ \pm}\right)$. Further, $M$ is the centralizer of $A$ in $G(\bmod A)$. $(M$ has the structure $M=M_{d} M_{r}$, where $M_{d}$ is a finite group, $M_{r}$ is reductive with the same Lie algebra $\mathscr{M}$ as $M$.) Then $P^{ \pm}=M A N^{ \pm}$are called parabolic subgroups of $G$.

The importance of the parabolic subgroups comes from the fact that the representations induced from them generate all (admissible) irreducible representations of $G$ [四], [䧃].

Let $P=M A N$ be an arbitrary parabolic subgroup, $\left(P=P^{+}, N=N^{+}\right.$, or $P=P^{-}, N=N^{-}$is specified by convenience). Let $\mu$ fix a finite-dimensional representation $D^{\mu}$ of $M$ on a space $V_{\mu}$. Let $v$ be a character of $A, v \in \mathscr{A}^{*}$. 
We call the induced representation $\chi=\operatorname{Ind}_{P}^{G}(\mu \otimes \boldsymbol{v} \otimes 1)$ an elementary representation of $G$. Consider the space of functions

$$
\mathscr{C}_{\chi}=\left\{\mathscr{F} \in C^{\infty}\left(G, V_{\mu}\right) \mid \mathscr{F}(\text { gman })=e^{v(H)} \cdot D^{\mu}\left(m^{-1}\right) \mathscr{F}(g)\right\}
$$

where $g \in G, m \in M, a=\exp (H) \in A, H \in \mathscr{A}, n \in N$. The special property of the functions of $\mathscr{C}_{\chi}$ is called right covariance.

Then the elementary representation (ER) $\mathscr{T} \chi$ acts in $\mathscr{C}_{\chi}$ as the left regular representation (LRR), by:

$$
\left(\mathscr{T}^{\chi}(g) \mathscr{F}\right)\left(g^{\prime}\right)=\mathscr{F}\left(g^{-1} g^{\prime}\right), \quad g, g^{\prime} \in G
$$

An important ingredient in our considerations is the existence of a Verma module $V^{\Lambda}$ over $\mathscr{G} \mathbb{C}$ associated to each elementary representation $\chi$, so that the weight $\Lambda=\Lambda(\chi) \in\left(\mathscr{H}^{\mathbb{C}}\right)^{*}$, $\left(\mathscr{H}^{\mathbb{C}}\right.$ is a Cartan subalgebra of $\left.\mathscr{G}^{\mathbb{C}}\right)$ is determined uniquely from $\chi$ [目].

We recall that a Verma module is a highest weight module $V^{\Lambda}$ with highest weight $L$, such that $V^{\Lambda} \cong U\left(\mathscr{G}_{-}^{\mathbb{C}}\right) v_{0}$, where $v_{0}$ is the highest weight vector, $U\left(\mathscr{G}_{-}^{\mathbb{C}}\right)$ is the universal enveloping algebra of $\mathscr{G}_{-}^{\mathbb{C}}$.

Generically, Verma modules are irreducible, however, we shall be mostly interested in the reducible ones since these are relevant for the construction of invariant differential operators. We recall the Bernstein-Gel'fand-Gel'fand criterion [G] according to which the Verma module $V^{\Lambda}$ is reducible iff

$$
\left\langle\Lambda+\rho, \beta^{\vee}\right\rangle=m
$$

holds for some $\beta \in \Delta^{+}, m \in \mathbb{N}$, where $\Delta^{+}$denotes the positive roots of the root system $\left(\mathscr{G}^{\mathbb{C}}, \mathscr{H}^{\mathbb{C}}\right)$, $\rho$ is half the sum of the positive roots $\Delta^{+}$.

Whenever ([L.5) is fulfilled there exists [6] in $V^{\Lambda}$ a unique vector $v_{s}$, called singular vector, such that $v_{s} \neq \mathbb{C} v_{0}$ and it has the properties of a highest weight vector with shifted weight $\Lambda-m \beta$ :

$$
\begin{aligned}
& \hat{X} v_{s}=(\Lambda-m \beta)(X) \cdot v_{s}, \quad X \in \mathscr{H}^{\mathbb{C}} \\
& \hat{X} v_{s}=0, \quad X \in \mathscr{G}_{+}^{\mathbb{C}}
\end{aligned}
$$

The general structure of a singular vector is [D]:

$$
v_{s}=P_{m \beta}\left(X_{1}^{-}, \ldots, X_{\ell}^{-}\right) v_{0}
$$

where $P_{m \beta}$ is a homogeneous polynomial in its variables of degrees $m k_{i}$, where $k_{i} \in \mathbb{Z}_{+}$come from the decomposition of $\beta$ into simple roots: $\beta=\sum k_{i} \alpha_{i}, \alpha_{i} \in \Delta_{S}$, the system of simple roots, $X_{j}^{-}$are the root vectors for $-\alpha_{j}, \alpha_{j}$ are the simple roots, $\ell=\operatorname{rank} \mathscr{G} \mathbb{C}$.

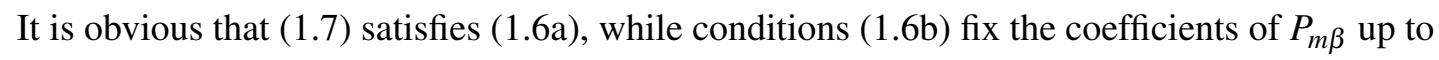
an overall multiplicative nonzero constant.

Now we are in a position to define the differential intertwining operators, corresponding to the singular vectors. Let the signature $\chi$ of an ER be such that the corresponding $\Lambda=\Lambda_{\chi}$ satisfies ([L.5) for some $\beta \in \Delta^{+}$and some $m \in \mathbb{N}$. Then there exists an intertwining differential operator []]

$$
\mathscr{D}_{m \beta}: \tilde{\mathscr{C}}_{\chi} \longrightarrow \tilde{\mathscr{C}}_{\chi^{\prime}}
$$


where $\chi^{\prime}$ is such that $\Lambda^{\prime}=\Lambda_{\chi^{\prime}}^{\prime}=\Lambda-m \beta$.

The important fact is that $([\mathbb{L} \cdot)$ is explicitly given by [[]]

$$
\mathscr{D}_{m \beta} \varphi(g)=P_{m \beta}\left(\hat{X}_{1}^{-}, \ldots, \hat{X}_{\ell}^{-}\right) \varphi(g)
$$

where $P_{m \beta}$ is the same polynomial as in $\left.(\mathbb{L}] \mathbb{Z}\right)$ and $\hat{X}_{j}^{-}$denotes the right action.

One important simplification is that in order to check the intertwining properties of the op-

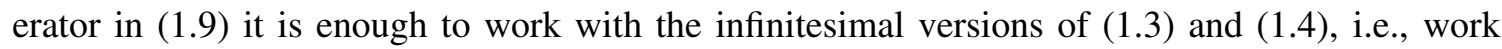
with representations of the Lie algebra. Thus, also in the quantum group setting we work with representations of quantum algebras.

\section{The matrix quantum group $\mathrm{GL}_{q}(\mathrm{n})$ and the dual quantum algebra}

Let us consider an $n \times n$ quantum matrix $M$ with non-commuting matrix elements $a_{i j}$, $1 \leq i, j \leq n$. The matrix quantum group $\mathscr{A}_{g}=G L_{q}(n), q \in \mathbb{C}$, is generated by the matrix elements $a_{i j}$ with the following commutation relations [四] $\left(\lambda=q-q^{-1}\right)$ :

$$
\begin{aligned}
& a_{i \ell} a_{i j}=q a_{i j} a_{i \ell}, \quad \ell>j, \quad a_{k j} a_{i j}=q a_{i j} a_{k j}, \quad k>i \\
& a_{k j} a_{i \ell}=a_{i \ell} a_{k j}, \quad k>i, \ell>j, \quad a_{i j} a_{k \ell}=a_{k \ell} a_{i j}-\lambda a_{i \ell} a_{k j}, \quad k>i, \ell>j
\end{aligned}
$$

Considered as a bialgebra, it has the following comultiplication $\delta_{\mathscr{A}}$ and counit $\varepsilon_{\mathscr{A}}$ :

$$
\delta_{\mathscr{A}}\left(a_{i j}\right)=\sum_{k=1}^{n} a_{i k} \otimes a_{k j}, \quad \varepsilon_{\mathscr{A}}\left(a_{i j}\right)=\delta_{i j}
$$

This algebra has determinant $D$ given by:

$$
D=\sum_{\rho \in S_{n}} \varepsilon(\rho) a_{1, \rho(1)} \ldots a_{n, \rho(n)}=\sum_{\rho \in S_{n}} \varepsilon(\rho) a_{\rho(1), 1} \ldots a_{\rho(n), n}
$$

where summations are over all permutations $\rho$ of $\{1, \ldots, n\}$ and the quantum signature is:

$$
\varepsilon(\rho)=\prod_{\substack{j<k \\ \rho(j)>\rho(k)}}\left(-q^{-1}\right)
$$

The determinant obeys:

$$
\delta_{\mathscr{A}}(D)=D \otimes D, \quad \varepsilon_{\mathscr{A}}(D)=1, \quad a_{i k} D=D a_{i k}
$$

Further, if $D \neq 0$ one extends the algebra by an element $D^{-1}$ which obeys:

$$
D D^{-1}=D^{-1} D=1_{\mathscr{A}}
$$

Then one can introduce the antipode in $G L_{q}(n)$ which is omitted for the lack of space.

Next we introduce a basis of $G L_{q}(n)$ which consists of monomials

$$
\begin{gathered}
f_{\bar{m}, \bar{\ell}, \bar{n}}=\left(a_{21}\right)^{m_{21}} \ldots\left(a_{n, n-1}\right)^{m_{n, n-1}} \times\left(a_{11}\right)^{\ell_{1}} \ldots\left(a_{n n}\right)^{\ell_{n}} \times\left(a_{n-1, n}\right)^{n_{n-1, n}} \ldots\left(a_{12}\right)^{n_{12}}, \\
f_{\overline{0}, \overline{0}, \overline{0}}=1_{\mathscr{A}_{g}}
\end{gathered}
$$


where $\bar{m}, \bar{\ell}, \bar{n}$ denote the sets $\left\{m_{i}\right\},\left\{\ell_{i j}\right\},\left\{n_{i j}\right\}$, resp., $m_{i}, \ell_{i j}, n_{i j} \in \mathbb{Z}_{+}$and we use the so-called normal ordering of the elements $a_{i j}$.

The dual algebra of $G L_{q}(n)$ is $\mathscr{U}_{g}=U_{q}(s l(n)) \otimes U_{q}(\mathscr{Z})$, where $U_{q}(\mathscr{Z})$ is central in $\mathscr{U}_{g}$. We denote the Chevalley generators of $s l(n)$ by $H_{i}, X_{i}^{ \pm}, i=1, \ldots, n-1$. Then we take for the 'Chevalley' generators of $\mathscr{U}=U_{q}(s l(n)): k_{i}=q^{H_{i} / 2}, k_{i}^{-1}=q^{-H_{i} / 2}, X_{i}^{ \pm}, i=1, \ldots, n-1$, with the following algebra relations:

$$
\begin{aligned}
& k_{i} k_{j}=k_{j} k_{i}, \quad k_{i} k_{i}^{-1}=k_{i}^{-1} k_{i}=1_{\mathscr{U}_{g}}, \quad k_{i} X_{j}^{ \pm}=q^{ \pm c_{i j}} X_{j}^{ \pm} k_{i} \\
& {\left[X_{i}^{+}, X_{j}^{-}\right]=\delta_{i j}\left(k_{i}^{2}-k_{i}^{-2}\right) / \lambda} \\
& \left(X_{i}^{ \pm}\right)^{2} X_{j}^{ \pm}-[2]_{q} X_{i}^{ \pm} X_{j}^{ \pm} X_{i}^{ \pm}+X_{j}^{ \pm}\left(X_{i}^{ \pm}\right)^{2}=0, \quad|i-j|=1 \\
& {\left[X_{i}^{ \pm}, X_{j}^{ \pm}\right]=0,|i-j| \neq 1}
\end{aligned}
$$

where $c_{i j}$ is the Cartan matrix of $s l(n)$, and coalgebra relations :

$$
\begin{aligned}
\delta_{\mathscr{U}}\left(k_{i}^{ \pm}\right) & =k_{i}^{ \pm} \otimes k_{i}^{ \pm}, \quad \delta_{\mathscr{U}}\left(X_{i}^{ \pm}\right)=X_{i}^{ \pm} \otimes k_{i}+k_{i}^{-1} \otimes X_{i}^{ \pm} \\
\varepsilon_{\mathscr{U}}\left(k_{i}^{ \pm}\right) & =1, \quad \varepsilon_{\mathscr{U}}\left(X_{i}^{ \pm}\right)=0 \\
\gamma_{\mathscr{U}}\left(k_{i}\right) & =k_{i}^{-1}, \quad \gamma_{\mathscr{U}}\left(X_{i}^{ \pm}\right)=-q^{ \pm 1} X_{i}^{ \pm}
\end{aligned}
$$

where $k_{i}^{+}=k_{i}, k_{i}^{-}=k_{i}^{-1}$. Further, we denote the generator of $\mathscr{Z}$ by $H$ and the generators of $U_{q}(\mathscr{Z})$ by $k=q^{H / 2}, k^{-1}=q^{-H / 2}, k k^{-1}=k^{-1} k=1 \mathscr{U}_{g}$. The generators $k, k^{-1}$ commute with the generators of $\mathscr{U}$, and their coalgebra relations are as those of any $k_{i}$.

The bilinear form giving the duality between $\mathscr{U}_{g}$ and $\mathscr{A}_{g}$ is given by [ [8]]:

$$
\begin{aligned}
& \left\langle k_{i}, a_{j \ell}\right\rangle=\delta_{j \ell} q^{\left(\delta_{i j}-\delta_{i, j+1}\right) / 2},\left\langle k, a_{j \ell}\right\rangle=\delta_{j \ell} q^{1 / 2} \\
& \left\langle X_{i}^{+}, a_{j \ell}\right\rangle=\delta_{j+1, \ell} \delta_{i j}, \quad\left\langle X_{i}^{-}, a_{j \ell}\right\rangle=\delta_{j-1, \ell} \delta_{i \ell} \\
& \left\langle y, 1_{\mathscr{A}_{g}}\right\rangle=\varepsilon_{\mathscr{U}_{g}}(y)
\end{aligned}
$$

The pairing between arbitrary elements of $\mathscr{U}_{g}$ and $f$ follows then from the properties of the duality pairing.

\section{Representations of $\mathscr{U}_{g}$ and $\mathscr{U}$}

We begin by defining two actions of the dual algebra $\mathscr{U}_{g}$ on the basis (2.7) of $\mathscr{A}_{g}$.

First we introduce the left regular representation of $\mathscr{U}_{g}$ for which in the $q=1$ case we need the infinitesimal version of :

$$
\pi(Y) M=Y^{-1} M, \quad Y, M \in G L(n)
$$

Explicitly, we define the action of $\mathscr{U}_{g}$ on $\mathscr{A}_{g}$ as follows (cf. also (따)):

$$
\pi(y) a_{i \ell} \doteq\left(F\left(\gamma_{\mathscr{U}}^{0}(y)\right) M\right)_{i \ell}=\sum_{j} F\left(\gamma_{\mathscr{U}}^{0}(y)\right)_{i j} a_{j \ell}=\sum_{j}\left\langle\gamma_{\mathscr{U}}^{0}(y), a_{i j}\right\rangle a_{j \ell}
$$

where $y$ denotes the generators of $\mathscr{U}_{g}$ and $\gamma_{\mathscr{U}}^{0}(y)$ is the antipode action for $q=1$.

In order to derive the action of $\pi(y)$ on arbitrary elements of the basis (2.7), we use the twisted derivation rule consistent with the coproduct and the representation structure, namely, we take: 
$\pi(y) \varphi \psi=\pi\left(\delta_{\mathscr{U}_{g}}^{\prime}(y)\right)(\varphi \otimes \psi)$, where $\delta_{\mathscr{U}_{g}}^{\prime}=\sigma \circ \delta_{\mathscr{U}_{g}}$ is the opposite coproduct, $(\sigma$ is the permutation operator). Thus, we have:

$$
\begin{aligned}
& \pi\left(k_{i}\right) \varphi \psi=\pi\left(k_{i}\right) \varphi \cdot \pi\left(k_{i}\right) \psi, \quad \pi(k) \varphi \psi=\pi(k) \varphi \cdot \pi(k) \psi \\
& \pi\left(X_{i}^{ \pm}\right) \varphi \psi=\pi\left(X_{i}^{ \pm}\right) \varphi \cdot \pi\left(k_{i}^{-1}\right) \psi+\pi\left(k_{i}\right) \varphi \cdot \pi\left(X_{i}^{ \pm}\right) \psi
\end{aligned}
$$

Analogously, we introduce the right action for which in the classical case one needs the infinitesimal counterpart of :

$$
\pi_{R}(Y) M=M Y, \quad Y, M \in G L(n)
$$

Thus, we define the right action of $\mathscr{U}_{g}$ as follows:

$$
\pi_{R}(y) a_{i \ell}=(M F(y))_{i \ell}=\sum_{j} a_{i j} F(y)_{j \ell}=\sum_{j} a_{i j}\left\langle y, a_{j \ell}\right\rangle
$$

where $y$ denotes the generators of $\mathscr{U}_{g}$.

The twisted derivation rule is now given by $\pi_{R}(y) \varphi \psi=\pi_{R}\left(\delta_{\mathscr{U}_{g}}(y)\right)(\varphi \otimes \psi)$, i.e.,

$$
\begin{aligned}
& \pi_{R}\left(k_{i}\right) \varphi \psi=\pi_{R}\left(k_{i}\right) \varphi \cdot \pi_{R}\left(k_{i}\right) \psi, \quad \pi_{R}(k) \varphi \psi=\pi_{R}(k) \varphi \cdot \pi_{R}(k) \psi \\
& \pi_{R}\left(X_{i}^{ \pm}\right) \varphi \psi=\pi_{R}\left(X_{i}^{ \pm}\right) \varphi \cdot \pi_{R}\left(k_{i}\right) \psi+\pi_{R}\left(k_{i}^{-1}\right) \varphi \cdot \pi_{R}\left(X_{i}^{ \pm}\right) \psi
\end{aligned}
$$

Let us now introduce the elements $\varphi$ as formal power series of the basis ([2.]):

$$
\varphi=\sum_{\bar{m}, \bar{\ell}, \bar{n} \in \mathbb{Z}_{+}} \mu_{\bar{m}, \bar{\ell}, \bar{n}} f_{\bar{m}, \bar{\ell}, \bar{n}}
$$

As in the classical case the left and right actions commute, and as in [[]] we shall use the right covariance to reduce the left regular representation. In particular, we require the right action to mimic properties of a highest weight module, i.e., annihilation by the raising generators $X_{i}^{+}$and scalar action by the (exponents of the) Cartan operators $k_{i}, k$. However, first we have to make a change of basis using the $q$-analogue of the classical Gauss decomposition. For this we have to suppose that the principal minor determinants of $M$ :

$$
D_{m}=\sum_{\rho \in S_{m}} \varepsilon(\rho) a_{1, \rho(1)} \ldots a_{m, \rho(m)}=\sum_{\rho \in S_{m}} \varepsilon(\rho) a_{\rho(1), 1} \ldots a_{\rho(m), m}, \quad m \leq n
$$

are invertible; note that $D_{n}=D, D_{n-1}=A_{n n}$.

Further, for the ordered sets $I=\left\{i_{1}<\cdots<i_{r}\right\}$ and $J=\left\{j_{1}<\cdots<j_{r}\right\}$, let $\xi_{J}^{I}$ be the $r$-minor determinant with respect to rows $I$ and columns $J$ such that

$$
\xi_{J}^{I}=\sum_{\rho \in S_{r}} \varepsilon(\rho) a_{i_{\rho(1)} j_{1}} \cdots a_{i_{\rho(r)} j_{r}}
$$

Note that $\xi_{1 \cdots i}^{1 \cdots i}=D_{i}$. Then we have $(i, j, \ell=1, \ldots, n)$ :

$$
a_{i \ell}=\sum_{j} \tilde{Y}_{i j} D_{j j} Z_{j \ell}, \tilde{Y}_{i j}=\xi_{1 \cdots j}^{1 \cdots j-1 i} D_{j}^{-1}, D_{j j}=D_{j} D_{j-1}^{-1}, Z_{j \ell}=D_{j}^{-1} \xi_{1 \cdots j-1 \ell}^{1 \cdots j}
$$


$\tilde{Y}_{i j}=0$ for $i<j, Z_{j \ell}=0$ for $j>\ell, D_{0} \equiv 1_{\mathscr{A}_{g}}$. Then $\tilde{Y}_{i j}, i>j$, may be regarded as a $q$-analogue of local coordinates of the $q$ - deformed flag manifold $G L(n) / D Z$, while $Z_{j \ell}, j<\ell$, may be regarded as a $q$-analogue of local coordinates of the $q$ - deformed flag manifold $\tilde{Y} D \backslash G L(n)$.

Clearly, we can replace the basis (D.]) of $\mathscr{A}_{g}$ with a basis in terms of $\tilde{Y}_{i \ell}, i>\ell, D_{\ell}, Z_{i \ell}, i<\ell$. (Note that $\tilde{Y}_{i i}=Z_{i i}=1_{\mathscr{A g}_{g}}$.) Thus, we consider formal power series:

$$
\tilde{\varphi}=\sum_{\substack{\bar{m}, \bar{n} \in \mathbb{Z}_{+} \\ \ell \in \mathbb{Z}}} \mu_{\bar{\ell}, \bar{m}, \bar{n}}^{\prime}\left(\tilde{Y}_{21}\right)^{m_{21}} \ldots\left(\tilde{Y}_{n, n-1}\right)^{m_{n, n-1}}\left(D_{1}\right)^{\ell_{1}} \ldots\left(D_{n}\right)^{\ell_{n}}\left(Z_{n-1, n}\right)^{n_{n-1, n}} \ldots\left(Z_{12}\right)^{n_{12}}
$$

Now, let us impose right covariance with respect to $X_{i}^{+}$:

$$
\pi_{R}\left(X_{i}^{+}\right) \tilde{\varphi}=0
$$

This is fulfilled automatically for $D_{j}$ and $\tilde{Y}_{j \ell}$, but not for $Z_{j \ell}$, which simply means that our functions $\tilde{\varphi}$ do not depend on $Z_{j \ell}$. Thus, the functions obeying (B.12) are:

$$
\tilde{\varphi}=\sum_{\bar{\ell} \in \mathbb{Z}, \bar{m} \in \mathbb{Z}_{+}} \mu_{\bar{\ell}, \bar{m}}\left(\tilde{Y}_{21}\right)^{m_{21}} \ldots\left(\tilde{Y}_{n, n-1}\right)^{m_{n, n-1}}\left(D_{1}\right)^{\ell_{1}} \ldots\left(D_{n}\right)^{\ell_{n}}
$$

Next, we impose right covariance with respect to $k_{i}, k$ :

$$
\pi_{R}\left(k_{i}\right) \tilde{\varphi}=q^{r_{i} / 2} \tilde{\varphi}, \quad \pi_{R}(k) \tilde{\varphi}=q^{\hat{r} / 2} \tilde{\varphi}
$$

As a consequence we obtain that the powers of $D_{i}$ in (B.D3) are fixed: $\ell_{i}=r_{i}$, for $i<n, \sum_{j=1}^{n} j \ell_{j}=$ $\hat{r}$. This means that $r_{i}, \hat{r} \in \mathbb{Z}$ and that there is no summation in $\ell_{i}$, also $\ell_{n}=\left(\hat{r}-\sum_{j=1}^{n-1} j r_{j}\right) / n \equiv \tilde{r}$.

Thus, the reduced functions obeying (B.12) and (B.14) are:

$$
\tilde{\varphi}=\sum_{\bar{m} \in \mathbb{Z}_{+}} \mu_{\bar{m}}\left(\tilde{Y}_{21}\right)^{m_{21}} \ldots\left(\tilde{Y}_{n, n-1}\right)^{m_{n, n-1}}, \quad\left(D_{1}\right)^{r_{1}} \ldots\left(D_{n-1}\right)^{r_{n-1}}\left(D_{n}\right)^{\tilde{r}}
$$

Next we derive the $\mathscr{U}_{g}$ - action $\pi$ on $\tilde{\varphi}$. First, we notice that $\mathscr{U}$ acts trivially on $D_{n}=D$ :

$$
\pi\left(X_{i}^{ \pm}\right) D=0, \quad \pi\left(k_{i}\right) D=D
$$

Then we note:

$$
\pi(k) D_{j}=q^{-j / 2} D_{j}, \quad \pi(k) \tilde{Y}_{j \ell}=\tilde{Y}_{j \ell}
$$

from which follows:

$$
\pi(k) \tilde{\varphi}=q^{-\hat{r} / 2} \tilde{\varphi}
$$

Thus, the action of $\mathscr{U}$ involves only the parameters $r_{i}, i<n$, while the action of $U_{q}(\mathscr{Z})$ involves only the parameter $\hat{r}$. Thus we can consistently also from the representation theory point of view restrict to the matrix quantum group $S L_{q}(n)$, i.e., we set:

$$
D=D^{-1}=1 \mathscr{A g}_{g}
$$

Then the dual algebra is $\mathscr{U}=U_{q}(s l(n))$. This is justified as in the $q=1$ case [వ] since for our considerations only the semisimple part of the algebra is important. 
Thus, the reduced functions for the $\mathscr{U}$ action are:

$$
\begin{aligned}
\tilde{\varphi}(\bar{Y}, \bar{D}) & =\sum_{\bar{m} \in \mathbb{Z}_{+}} \mu_{\bar{m}}\left(\tilde{Y}_{21}\right)^{m_{21}} \ldots\left(\tilde{Y}_{n, n-1}\right)^{m_{n, n-1}}\left(D_{1}\right)^{r_{1}} \ldots\left(D_{n-1}\right)^{r_{n-1}}= \\
& =\hat{\varphi}(\bar{Y})\left(D_{1}\right)^{r_{1}} \ldots\left(D_{n-1}\right)^{r_{n-1}}
\end{aligned}
$$

where $\bar{Y}, \bar{D}$ denote the variables $\tilde{Y}_{i l}, i>\ell, D_{i}, i<n$.

From (B.20) it is clear that the parameters $r_{i}$ indeed characterize the representation of $\mathscr{U}$. Furthermore, we can introduce the restricted functions $\hat{\varphi}(\bar{Y})$ by the formula which is prompted in (B.20b):

$$
\hat{\varphi}(\bar{Y}) \doteq \tilde{\varphi}\left(\bar{Y}, D_{j}=1_{\mathscr{A}_{g}}\right)=\sum_{\bar{m} \in \mathbb{Z}_{+}} \mu_{\bar{m}}\left(\tilde{Y}_{21}\right)^{m_{21}} \ldots\left(\tilde{Y}_{n, n-1}\right)^{m_{n, n-1}}
$$

We have defined the representations $\hat{\pi}_{\bar{r}}$ for $r_{i} \in \mathbb{Z}$. However, notice that we can consider the restricted functions $\hat{\varphi}(\bar{Y})$ for arbitrary complex $r_{i}$. We shall make these extension from now on, since this gives the same set of representations for $U_{q}(s l(n))$ as in the case $q=1$.

For the more compact exposition of the representation formulae we shall need below also the following operators (corresponding to each $\tilde{Y}_{j \ell}$ ) :

$$
\begin{aligned}
& \hat{M}_{j \ell} \hat{\varphi}(\bar{Y})=\sum_{\bar{m} \in \mathbb{Z}_{+}} \mu_{\bar{m}} \hat{M}_{j \ell} \tilde{f}_{\bar{m}}, \quad \hat{M}_{j \ell} \tilde{f}_{\bar{m}}=\left(\tilde{Y}_{21}\right)^{m_{21}} \ldots\left(\tilde{Y}_{j \ell}\right)^{m_{j \ell}+1} \ldots\left(\tilde{Y}_{n, n-1}\right)^{m_{n, n-1}} \\
& \tilde{f}_{\bar{m}}=\left(\tilde{Y}_{21}\right)^{m_{21}} \ldots\left(\tilde{Y}_{n, n-1}\right)^{m_{n, n-1}}, \\
& T_{j \ell} \hat{\varphi}(\bar{Y})=\sum_{\bar{m} \in \mathbb{Z}_{+}} \mu_{\bar{m}} T_{j \ell} \tilde{f}_{\bar{m}}, \quad T_{j \ell} \tilde{f}_{\bar{m}}=q^{m_{j \ell}} \tilde{f}_{\bar{m}}
\end{aligned}
$$

Using this we define the $q$-difference operators by:

$$
\hat{\mathscr{D}}_{j \ell} \hat{\varphi}(\bar{Y})=\frac{1}{\lambda} \hat{M}_{j \ell}^{-1}\left(T_{j \ell}-T_{j \ell}^{-1}\right) \hat{\varphi}(\bar{Y})
$$

from which follows:

$$
\hat{\mathscr{D}}_{j \ell} \tilde{f}_{\bar{m}}=\left[m_{j \ell}\right]_{q}\left(\tilde{Y}_{21}\right)^{m_{21}} \ldots\left(\tilde{Y}_{j \ell}\right)^{m_{j \ell}-1} \ldots\left(\tilde{Y}_{n, n-1}\right)^{m_{n, n-1}}
$$

Of course, for $q \rightarrow 1$ we have $\hat{\mathscr{D}}_{j \ell} \rightarrow \partial_{Y_{j \ell}} \equiv \partial / \partial Y_{j \ell}$. (Note that the above operators for different variables commute, i.e., with these we have actually passed to commuting variables.)

\section{The case of $\mathbf{U}_{q}(\mathbf{s}(\mathbf{4}))$}

Here we consider in more detail the case $n=4$. It is convenient (also for the comparison with the $q=1$ case) to make the following change of variables:

$$
\begin{aligned}
& Y_{31}=\tilde{Y}_{31}-q \tilde{Y}_{21} \tilde{Y}_{32}, \quad Y_{41}=\tilde{Y}_{41}-q \tilde{Y}_{21} \tilde{Y}_{42}, \\
& Y_{21}=-q \tilde{Y}_{21}, \quad Y_{43}=q \tilde{Y}_{43}, \quad Y_{i j}=\tilde{Y}_{i j}, \quad \text { for }(i j)=(32),(42)
\end{aligned}
$$

For the commutation properties we have:

$$
\begin{aligned}
& Y_{i \ell} Y_{i j}=q^{1-\delta_{\ell 2}} Y_{i j} Y_{i \ell}, \quad 4 \geq i>\ell>j \geq 1 \\
& Y_{k j} Y_{i j}=q^{1-\delta_{i 2}} Y_{i j} Y_{k j}, \quad 4 \geq k>i>j \geq 1 \\
& Y_{41} Y_{32}=Y_{32} Y_{41}+\lambda Y_{31} Y_{42}, \\
& Y_{4 i} Y_{j 1}=Y_{j 1} Y_{4 i}, \quad(i j)=(23),(32), \\
& Y_{k i} Y_{i j}=q^{1-2 \delta_{i 3}} Y_{i j} Y_{k i}-(-1)^{\delta_{i 3}} \lambda Y_{k j}{ }^{\prime} \quad 4 \geq k>i>j \geq 1
\end{aligned}
$$


(each of (4.2/a,b,e) has four cases).

Note that for $q$ a phase $(|q|=1)$ the $q$ - deformed flag manifold in the $Y$ coordinates is invariant under the anti-linear anti-involution $\omega$ acting as:

$$
\omega\left(Y_{j \ell}\right)=Y_{5-\ell, 5-j}
$$

Thus it can be considered as a $q$ - deformed flag manifold of the quantum group $S U_{q}(2,2)$.

The restricted functions for the $\mathscr{U}$ action are (cf. (B.21)):

$$
\begin{gathered}
\hat{\varphi}(\bar{Y})=\sum_{i, j, k, \ell, m, n \in \mathbb{Z}_{+}} \mu_{i j k \ell m n} \hat{\varphi}_{i j k \ell m n} \\
\hat{\varphi}_{i j k \ell m n}=\left(Y_{21}\right)^{i}\left(Y_{31}\right)^{j}\left(Y_{32}\right)^{k}\left(Y_{41}\right)^{\ell}\left(Y_{42}\right)^{m}\left(Y_{43}\right)^{n}
\end{gathered}
$$

Recall that we consider the representations $\hat{\pi}_{\bar{r}}$ for arbitrary complex $r_{i}$ and we know from the general analysis of [2] that whenever some $m_{i}=r_{i}+1$ or $m_{i j}=m_{i}+\cdots+m_{j},(i<j)$ is a positive integer the representations are reducible and there exist invariant subspaces.

It will be convenient to use also the following notation for the coordinates of the flag manifold:

$$
\xi=Y_{21}, \quad x=Y_{31}, \quad u=Y_{32}, \quad w=Y_{41}, \quad y=Y_{42}, \quad \eta=Y_{43}
$$

\section{Intertwining operators}

The general prescription for finding the intertwining operators is as in the classical case (cf. also [四]). In order to apply this procedure we need the explicit action of $\pi_{R}\left(X_{i}^{-}\right)$on our functions. We have:

$$
\begin{aligned}
& \pi_{R}\left(X_{1}^{-}\right) \tilde{\varphi}_{i j k \ell m n}^{r_{1}, r_{2}, r_{3}}=-q^{i-j-k-\ell-m+\left(r_{1}-1\right) / 2}[i]_{q} \tilde{\varphi}_{i-1, j k \ell m n}^{r_{1}-2, r_{2}+1, r_{3}}+q^{\left(r_{1}-1\right) / 2}\left[r_{1}\right]_{q} \tilde{\varphi}_{i j k \ell m n}^{r_{1}, r_{2}, r_{3}} Z_{12} \\
& \pi_{R}\left(X_{2}^{-}\right) \tilde{\varphi}_{i j k \ell m n}^{r_{1}, r_{2}, r_{3}}=q^{2 k+\ell+m-n+\left(r_{2}-1\right) / 2}[j]_{q} \tilde{\varphi}_{i+1, j-1, k \ell m n}^{r_{1}+1, r_{2}-2, r_{3}+1}+ \\
& +q^{k+\ell+m-n+\left(r_{2}-3\right) / 2}[k]_{q} \tilde{\varphi}_{i j, k-1, \ell m n}^{r_{1}+1, r_{2}-2, r_{3}+1}+ \\
& +q^{k-j+2 m-n+\left(r_{2}-3\right) / 2}[\ell]_{q} \tilde{\varphi}_{i+1, j k, \ell-1, m, n+1}^{r_{1}+1, r_{2}-2, r_{3}+1}+ \\
& +q^{m-n+\left(r_{2}-5\right) / 2}[m]_{q} \tilde{\varphi}_{i j k \ell, m-1, n+1}^{r_{1}+1, r_{2}-2, r_{3}+1} \\
& -q^{2 m-n+\left(r_{2}-3\right) / 2} \lambda[k]_{q}[\ell]_{q} \tilde{\varphi}_{i, j+1, k-1, \ell-1, m, n+1}^{r_{1}+1, r_{2}-2, r_{3}+1}+ \\
& +q^{\left(r_{2}-1\right) / 2}\left[r_{2}\right]_{q} \tilde{\varphi}_{i j k \ell m n}^{r_{1}, r_{2}, r_{3}} Z_{23} \\
& \pi_{R}\left(X_{3}^{-}\right) \tilde{\varphi}_{i j k \ell m n}^{r_{1}, r_{2}, r_{3}}=q^{n+\left(r_{3}-1\right) / 2}[n]_{q} \tilde{\varphi}_{i j k \ell m, n-1}^{r_{1}, r_{2}+1, r_{3}-2}+q^{\left(r_{3}-1\right) / 2}\left[r_{3}\right]_{q} \tilde{\varphi}_{i j k \ell m n}^{r_{1}, r_{2}, r_{3}} Z_{34}
\end{aligned}
$$

where we have labelled the functions also with the representation parameters $r_{s}$. As in the classical case [四] the right action is taking out from the representation space $\mathscr{C}_{\bar{r}}$, and while some of the terms are functions from other representation spaces (depending on which $X_{s}^{-}$is acting), there are terms involving the $Z_{j \ell}$ variables which do not belong to any of our representation spaces. The terms with $Z_{j \ell}$ vanish exactly when $r_{s} \in \mathbb{Z}_{+}$and we take $\left(\pi_{R}\left(X_{s}^{-}\right)\right)^{m_{s}}$ [[2], $m_{s}=r_{s}+1$.

We also use $q$-difference operators (using notation (B.22), (B.23), (4.5])):

$$
\begin{aligned}
\hat{I}_{1}= & -q^{\left(r_{1}-1\right) / 2} \hat{\mathscr{D}}_{\xi} T_{\xi}\left(T_{x} T_{u} T_{w} T_{y}\right)^{-1} \\
\hat{I}_{2}= & q^{\left(r_{2}-3\right) / 2}\left(q \hat{M}_{\xi} \hat{\mathscr{D}}_{x} T_{u}+\hat{\mathscr{D}}_{u}+\hat{M}_{\xi} \hat{M}_{\eta} \hat{\mathscr{D}}_{w}\left(T_{x} T_{w}\right)^{-1} T_{y}+\right. \\
& \left.+q^{-1} \hat{M}_{\eta} \hat{\mathscr{D}}_{y}\left(T_{u} T_{w}\right)^{-1}-\lambda \hat{M}_{x} \hat{M}_{\eta} \hat{\mathscr{D}}_{u} \hat{\mathscr{D}}_{w}\left(T_{u} T_{w}\right)^{-1} T_{y}\right) T_{u} T_{w} T_{y} T_{\eta}^{-1}, \\
\hat{I}_{3}= & q^{\left(r_{3}-1\right) / 2} \hat{\mathscr{D}}_{\eta} T_{\eta}
\end{aligned}
$$


and we note (for $m_{s} \in \mathbb{N}$ ):

$$
\hat{I}_{s}^{m_{s}}=I_{s}^{m_{s}}=\left(\pi_{R}\left(X_{s}^{-}\right)\right)^{m_{s}}
$$

Let us consider now the intertwining operators corresponding to the two non-simple nonhighest roots $\alpha_{12}, \alpha_{23}$ which are realized when $m_{12} \in \mathbb{N}, m_{23} \in \mathbb{N}$, resp. In these cases the intertwining operators (up to an overall multiplicative constant) are given by :

$$
\begin{aligned}
I_{i j}^{m} & =\sum_{k=0}^{m} a_{k}\left(\pi_{R}\left(X_{i}^{-}\right)\right)^{m-k}\left(\pi_{R}\left(X_{j}^{-}\right)\right)^{m}\left(\pi_{R}\left(X_{i}^{-}\right)\right)^{k}, \quad m=m_{i j}, \quad(i j)=(12),(23), \\
a_{k} & =(-1)^{k} a \frac{\left[m_{i}\right]_{q}}{\left[m_{i}-k\right]_{q}}\left(\begin{array}{c}
m \\
k
\end{array}\right)_{q}, \quad k=0, \ldots, m, a \neq 0
\end{aligned}
$$

Let us illustrate the resulting intertwining operators in the cases $m_{12}=1, m_{23}=1$. We have (after a suitable renormalization) :

$$
\begin{aligned}
\left.I_{12}^{1}\right|_{r_{1}+r_{2}=-1} & =-\left[r_{1}\right]_{q} \pi_{R}\left(X_{1}^{-}\right) \pi_{R}\left(X_{2}^{-}\right)+\left[r_{1}+1\right]_{q} \pi_{R}\left(X_{2}^{-}\right) \pi_{R}\left(X_{1}^{-}\right)= \\
& =-\left[r_{1}\right]_{q} \hat{I}_{1} \hat{I}_{2}+\left[r_{1}+1\right]_{q} \hat{I}_{2} \hat{I}_{1} \\
\left.I_{23}^{1}\right|_{r_{2}+r_{3}=-1} & =-\left[r_{3}\right]_{q} \pi_{R}\left(X_{3}^{-}\right) \pi_{R}\left(X_{2}^{-}\right)+\left[r_{3}+1\right]_{q} \pi_{R}\left(X_{2}^{-}\right) \pi_{R}\left(X_{3}^{-}\right)= \\
& =-\left[r_{3}\right]_{q} \hat{I}_{3} \hat{I}_{2}+\left[r_{3}+1\right]_{q} \hat{I}_{2} \hat{I}_{3}
\end{aligned}
$$

\section{6. q - Minkowski space-time and q - Maxwell equations hierarchy from q - conformal invariance}

6.1. We start with the $q=1$ situation and we first write the Maxwell equations in an indexless formulation, trading the indices for two conjugate variables $z, \bar{z}$. This formulation has two advantages.

First, it is very simple, and in fact, just with the introduction of an additional parameter, we can describe a whole infinite hierarchy of equations, which we call the Maxwell hierarchy .

Second, we can easily identify the variables $z, \bar{z}$ and the four Minkowski coordinates with the six local coordinates of a flag manifold of $S U(2,2)$, or of $S L(4)$ with the appropriate conjugation. Thus, one may look at this as a nice example of unifying internal and external degrees of freedom.

Next we give the $q$ - analogs using the $U_{q}(s l(4))$ apparatus of Sections 4 and 5. Thus, we use $q$ - Minkowski coordinates as part of the appropriate $q$-deformed flag manifold. Using the corresponding representations and intertwiners of $U_{q}(s l(4))$ we can finally write down the infinite hierarchy of $q$ - Maxwell equations.

6.2. It is well known that Maxwell equations

$$
\partial^{\mu} F_{\mu v}=J_{v}, \quad \partial^{\mu *} F_{\mu v}=0
$$

or, equivalently

$$
\partial_{k} E_{k}=J_{0}, \quad \partial_{0} E_{k}-\varepsilon_{k \ell m} \partial_{\ell} H_{m}=J_{k}, \quad \partial_{k} H_{k}=0, \quad \partial_{0} H_{k}+\varepsilon_{k \ell m} \partial_{\ell} E_{m}=0
$$

where $E_{k} \equiv F_{k 0}, H_{k} \equiv(1 / 2) \varepsilon_{k \ell m} F_{\ell m}$, can be rewritten in the following manner:

$$
\partial_{k} F_{k}^{ \pm}=J_{0}, \quad \partial_{0} F_{k}^{ \pm} \pm i \varepsilon_{k \ell m} \partial_{\ell} F_{m}^{ \pm}=J_{k}
$$


where

$$
F_{k}^{ \pm} \equiv E_{k} \pm i H_{k}
$$

Not so well known is the fact that the eight equations in (6.3]) can be rewritten as two conjugate scalar equations in the following way:

$$
I^{+} F^{+}(z)=J(z, \bar{z}), \quad I^{-} F^{-}(\bar{z})=J(z, \bar{z})
$$

where

$$
\begin{gathered}
I^{+}=\bar{z} \partial_{+}+\partial_{v}-\frac{1}{2}\left(\bar{z} z \partial_{+}+z \partial_{v}+\bar{z} \partial_{\bar{v}}+\partial_{-}\right) \partial_{z} \\
I^{-}=z \partial_{+}+\partial_{\bar{v}}-\frac{1}{2}\left(\bar{z} z \partial_{+}+z \partial_{v}+\bar{z} \partial_{\bar{v}}+\partial_{-}\right) \partial_{\bar{z}} \\
x_{ \pm} \equiv x_{0} \pm x_{3}, \quad v \equiv x_{1}-i x_{2}, \quad \bar{v} \equiv x_{1}+i x_{2} \\
\partial_{ \pm} \equiv \partial / \partial x_{ \pm}, \quad \partial_{v} \equiv \partial / \partial v, \quad \partial_{\bar{v}} \equiv \partial / \partial \bar{v} \\
F^{+}(z) \equiv \quad \\
F^{-}(\bar{z}) \equiv \quad z^{2}\left(F_{1}^{+}+i F_{2}^{+}\right)-2 z F_{3}^{+}-\left(F_{1}^{+}-i F_{2}^{+}\right) \\
J(z, \bar{z}) \equiv \bar{z} z\left(J_{0}+J_{3}\right)+\bar{z}\left(J_{1}-i J_{2}\right)+z\left(J_{1}+i J_{2}\right)+\left(J_{0}-J_{3}\right)
\end{gathered}
$$

where we continue to suppress the $x_{\mu}$, resp., $x_{ \pm}, v, \bar{v}$, dependence in $F$ and $J$. (The conjugation mentioned above is standard and in our terms it is: $I^{+} \longleftrightarrow I^{-}, F^{+}(z) \longleftrightarrow F^{-}(\bar{z})$.)

It is easy to recover (6.3) from (6.5) - just note that both sides of each equation are first order polynomials in each of the two variables $z$ and $\bar{z}$, then comparing the independent terms in (6.5) one gets at once (6.3)).

Writing the Maxwell equations in the simple form (6.5) has also important conceptual meaning. The point is that each of the two scalar operators $I^{+}, I^{-}$is indeed a single object, namely it is

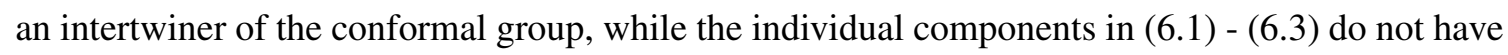
this interpretation. This is also the simplest way to see that the Maxwell equations are conformally invariant, since this is equivalent to the intertwining property.

Let us be more explicit. The physically relevant representations $T^{\chi}$ of the 4-dimensional conformal algebra $\operatorname{su}(2,2)$ may be labelled by $\chi=\left[n_{1}, n_{2} ; d\right]$, where $n_{1}, n_{2}$ are non-negative integers fixing finite-dimensional irreducible representations of the Lorentz subalgebra, (the dimension being $\left(n_{1}+1\right)\left(n_{2}+1\right)$ ), and $d$ is the conformal dimension (or energy). (In the literature these Lorentz representations are labelled also by $\left(j_{1}, j_{2}\right)=\left(n_{1} / 2, n_{2} / 2\right)$.) Then the intertwining properties of the operators in (6.6) are given by:

$$
\begin{aligned}
& I^{+}: C^{+} \longrightarrow C^{0}, \quad I^{+} \circ T^{+}=T^{0} \circ I^{+} \\
& I^{-}: C^{-} \longrightarrow C^{0}, \quad I^{-} \circ T^{-}=T^{0} \circ I^{-}
\end{aligned}
$$

where $T^{a}=T^{\chi^{a}}, a=0,+,-, C^{a}=C^{\chi^{a}}$ are the representation spaces, and the signatures are given explicitly by:

$$
\chi^{+}=[2,0 ; 2], \quad \chi^{-}=[0,2 ; 2], \quad \chi^{0}=[1,1 ; 3]
$$


as anticipated. Indeed, $\left(n_{1}, n_{2}\right)=(1,1)$ is the four-dimensional Lorentz representation, (carried by $J_{\mu}$ above), and $\left(n_{1}, n_{2}\right)=(2,0),(0,2)$ are the two conjugate three-dimensional Lorentz representations, (carried by $F_{k}^{ \pm}$above), while the conformal dimensions are the canonical dimensions of a current $(d=3)$, and of the Maxwell field $(d=2)$. We see that the variables $z, \bar{z}$ are related to the spin properties and we shall call them 'spin variables'. In general, a Lorentz spin-tensor $G(z, \bar{z})$ with signature $\left(n_{1}, n_{2}\right)$ is a polynomial in $z, \bar{z}$ of order $n_{1}, n_{2}$, resp.

We can illustrate the above occurrence with the following diagrams of intertwining operators:

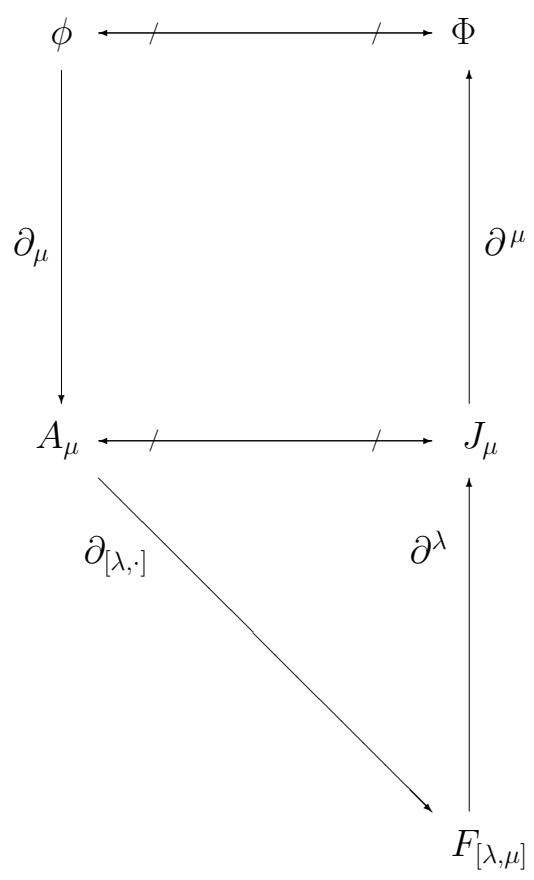

Fig. 1. Simplest example of diagram with conformal invariant operators (arrows are differential operators, dashed arrows are integral operators)

$\partial_{\mu}=\frac{\partial}{\partial x_{\mu}}, A_{\mu}$ electromagnetic potential, $\partial_{\mu} \phi=A_{\mu}^{0}$

$F$ electromagnetic field, $\partial_{[\lambda} A_{\mu]}=\partial_{\lambda} A_{\mu}-\partial_{\mu} A_{\lambda}=F_{\lambda \mu}^{0}$

$J_{\mu}$ electromagnetic current, $\partial^{\lambda} F_{\lambda \mu}=J_{\mu}^{0}, \partial^{\mu} J_{\mu}=\Phi^{0}$

superscript '0' indicates that the mapping is not onto 


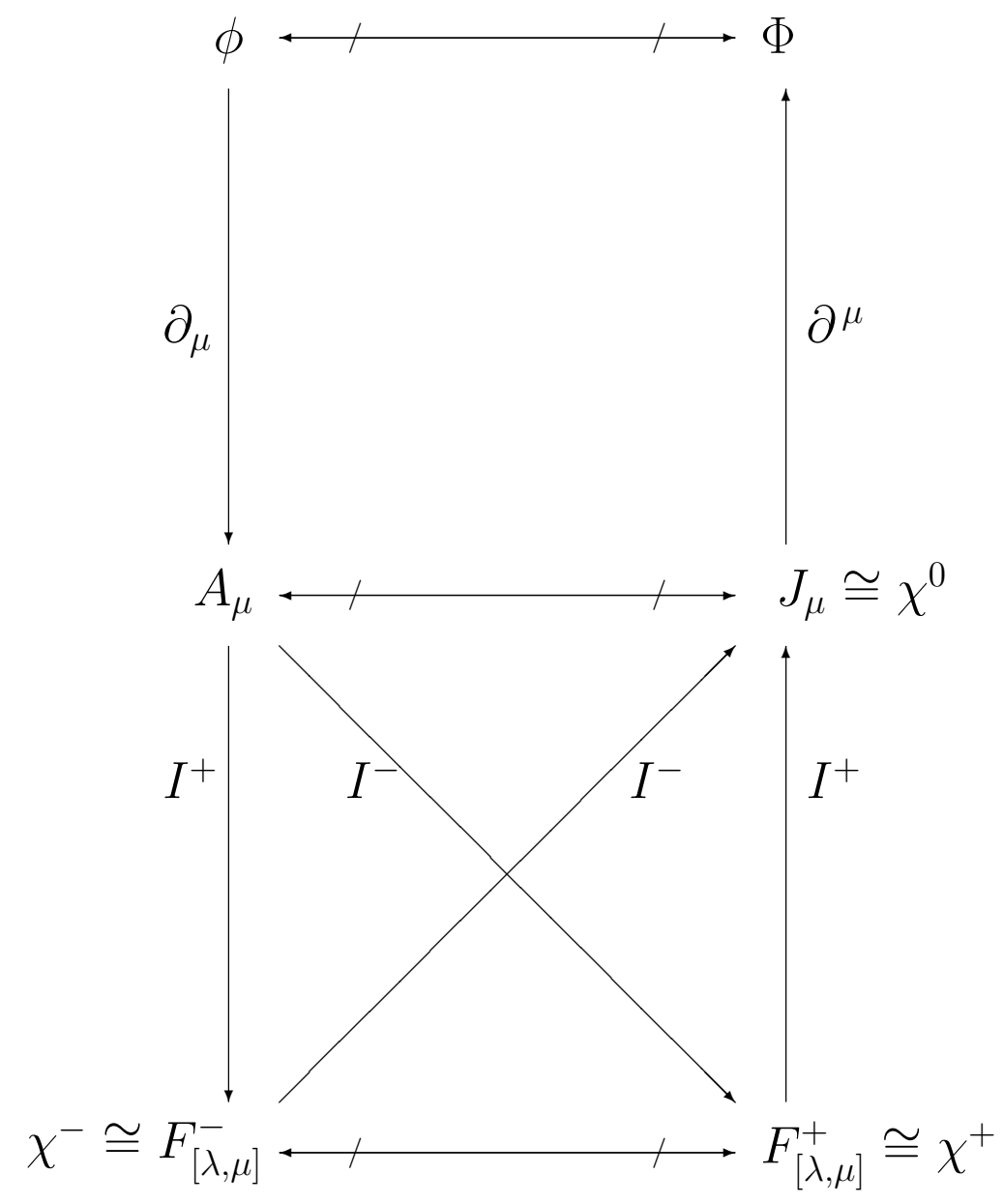

Fig. 2. More precise showing of the simplest example, using also notations from the text above 


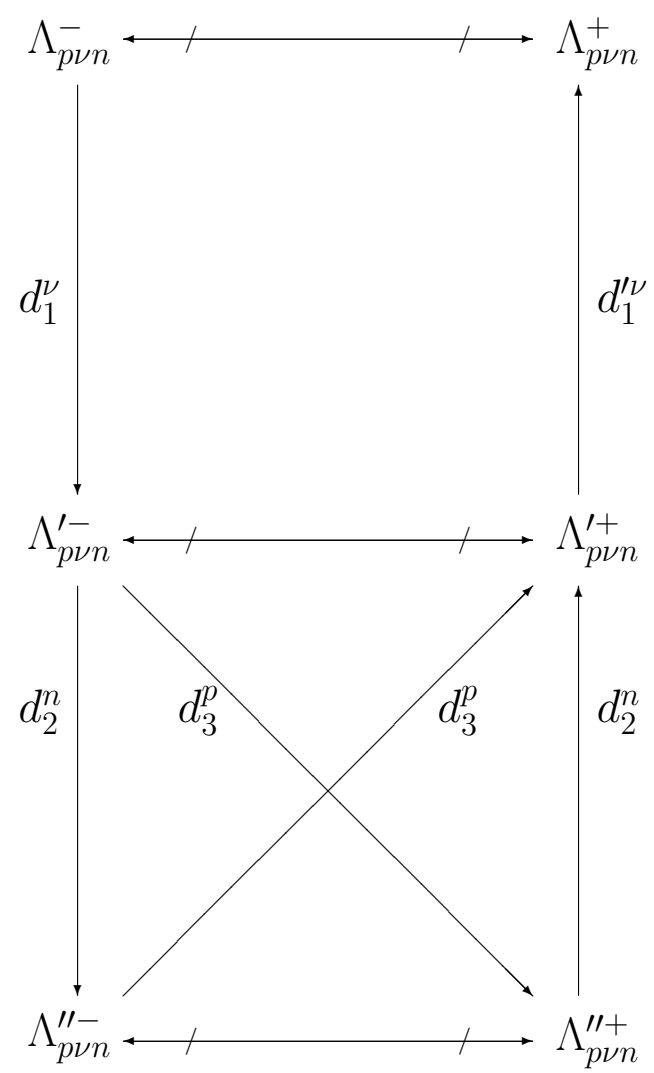

Fig. 3. The general classification of invariant differential operators valid for so $(4,2)$, so $(5,1)$ and $s o(3,3)$.

$p, \nu, n$ are three natural numbers, the shown simplest case is when $p=\nu=n=1$, $d_{1}^{\nu}$ is a linear differential operator of order $\nu$, similarly $d_{1}^{\prime \nu}, d_{2}^{n}, d_{3}^{p}$ 
From the last figure we can derive the fact that the Maxwell equations (6.9), (6.10) are part of an infinite hierarchy of couples of first order invariant equations (that would be obtained for $p=n=1)$. Explicitly, instead of (6.9), (6.10) we have [8]:

$$
\begin{array}{ll}
I_{v}^{+}: C_{v}^{+} \longrightarrow C_{v}^{0}, & I_{v}^{+} \circ T_{v}^{+}=T_{v}^{0} \circ I_{v}^{+} \\
I_{v}^{-}: C_{v}^{-} \longrightarrow C_{v}^{0}, & I_{v}^{-} \circ T_{v}^{-}=T_{v}^{0} \circ I_{v}^{-}
\end{array}
$$

where $T_{v}^{a}=T^{\chi_{v}^{a}}, C_{v}^{a}=C^{\chi_{v}^{a}}$, and the signatures are:

$$
\chi_{v}^{+}=[v+1, v-1 ; 2], \quad \chi_{v}^{-}=[v-1, v+1 ; 2], \quad \chi_{v}^{0}=[v, v ; 3], \quad v \in \mathbb{N}
$$

while instead of (6.5) we have:

$$
I_{v}^{+} F_{v}^{+}(z, \bar{z})=J_{v}(z, \bar{z}), \quad I_{v}^{-} F_{v}^{-}(z, \bar{z})=J_{v}(z, \bar{z})
$$

where

$$
\begin{array}{ll}
I_{v}^{+}=\frac{v+1}{2}\left(\bar{z} \partial_{+}+\partial_{v}\right)-\frac{1}{2}\left(\bar{z} z \partial_{+}+z \partial_{v}+\bar{z} \partial_{\bar{v}}+\partial_{-}\right) \partial_{z}, & v \in \mathbb{N} \\
I_{v}^{-}=\frac{v+1}{2}\left(z \partial_{+}+\partial_{\bar{v}}\right)-\frac{1}{2}\left(\bar{z} z \partial_{+}+z \partial_{v}+\bar{z} \partial_{\bar{v}}+\partial_{-}\right) \partial_{\bar{z}}, & v \in \mathbb{N}
\end{array}
$$

while $F_{v}^{+}(z, \bar{z}), F_{v}^{-}(z, \bar{z}), J_{v}(z, \bar{z})$, are polynomials in $z, \bar{z}$ of degrees $(v+1, v-1),(v-1, v+1)$, $(v, v)$, resp., as explained above.

Remark: If we want to use the notation with indices as in (6. ل. $)$, then $F_{v}^{+}(z, \bar{z})$ and $F_{v}^{-}(z, \bar{z})$ correspond to $F_{\lambda \mu, \alpha_{1}, \ldots, \alpha_{v-1}}$ which is antisymmetric in the indices $\lambda$, $\mu$, symmetric in $\alpha_{1}, \ldots, \alpha_{v-1}$, and traceless in every pair of indices, while $J_{v}(z, \bar{z})$ corresponds to $J_{\mu, \alpha_{1}, \ldots, \alpha_{v-1}}$ which is symmetric and traceless in every pair of indices. Note, however, that the analogs of (G. Would be much more complicated if one wants to write explicitly all components. The crucial advantage of (6.13) is that the operators $I_{V}^{ \pm}$are given just by a slight generalization of $I^{ \pm}=I_{1}^{ \pm} . \diamond$

We shall call the hierarchy of equations (6.53) the Maxwell hierarchy . The Maxwell equations are the zero member of this hierarchy.

To proceed further we rewrite (6.14) in the following form:

$$
I_{v}^{+}=\frac{1}{2}\left((v+1) I_{1} I_{2}-(v+2) I_{2} I_{1}\right), \quad I_{v}^{-}=\frac{1}{2}\left((v+1) I_{3} I_{2}-(v+2) I_{2} I_{3}\right)
$$

where

$$
I_{1} \equiv \partial_{z}, \quad I_{2} \equiv \bar{z} z \partial_{+}+z \partial_{v}+\bar{z} \partial_{\bar{v}}+\partial_{-}, \quad I_{3} \equiv \partial_{\bar{z}}
$$

We note in passing that group-theoretically the operators $I_{a}$ correspond to the three simple roots of the root system of $s l(4)$, while the operators $I_{v}^{ \pm}$correspond to the two non-simple non-highest roots [व].

This is the form that we generalize for the $q$ - deformed case. In fact, we can write at once the general form of the intertwining operators which follows from (5.5) (cf. also (5.2)) :

$$
{ }_{q} I_{v}^{+}=\frac{1}{2}\left([v+1]_{q} \hat{I}_{1} \hat{I}_{2}-[v+2]_{q} \hat{I}_{2} \hat{I}_{1}\right), \quad{ }_{q} I_{v}^{-}=\frac{1}{2}\left([v+1]_{q} \hat{I}_{3} \hat{I}_{2}-[v+2]_{q} \hat{I}_{2} \hat{I}_{3}\right)
$$

It is our task (using the previous Sections) to make this form explicit by introducing the appropriate variables and functions. 
6.3. The variables $x_{ \pm}, v, \bar{v}, z, \bar{z}$ have definite group-theoretical meaning, namely, they are six local coordinates on the flag manifold $\mathscr{Y}=S L(4) / B$, where $B$ is the Borel subgroup of $S L(4)$ consisting of all upper diagonal matrices. Under the natural conjugation (cf. also below) this is also a flag manifold of the conformal group $S U(2,2)$.

We know from Sections 3. and 4. what are the properties of the non-commutative coordinates on the $q$ - deformed $S L(4)$ flag manifold. We make the following identification:

$$
\begin{aligned}
& x_{+}=w=Y_{41}, \quad x_{-}=u=Y_{32}, \quad v=x=Y_{31}, \quad \bar{v}=y=Y_{42} \\
& z=\xi=Y_{21}, \quad \bar{z}=\eta=Y_{43}
\end{aligned}
$$

for the $q$-Minkowski space-time coordinates and for the spin coordinates, which we denote as their classical counterparts. Thus, we obtain for the commutation rules of the $q$-Minkowski space-time coordinates (cf. (4.2)) :

$$
x_{ \pm} v=q^{ \pm 1} v x_{ \pm}, \quad x_{ \pm} \bar{v}=q^{ \pm 1} \bar{v} x_{ \pm}, \quad x_{+} x_{-}-x_{-} x_{+}=\lambda v \bar{v}, \quad \bar{v} v=v \bar{v}
$$

As expected, relations (6.9) coincide with the commutation relations between the translation generators $P_{\mu}$ of the $q$-conformal algebra [Q].

It is also easy to notice that these relations are as the $G L_{q}(2)$ commutation relations [ $[$ ] , if we identify our coordinates with the standard $a, b, c, d$ generators of $G L_{q}(2)$ as follows:

$$
M=\left(\begin{array}{ll}
a & b \\
c & d
\end{array}\right)=\left(\begin{array}{cc}
x_{+} & v \\
\bar{v} & x_{-}
\end{array}\right)
$$

Thus, the $q$-Minkowski length is defined as the $G L_{q}(2) q$-determinant:

$$
\ell_{q} \doteq \operatorname{det}_{q} M=a d-q b c=x_{+} x_{-}-q \bar{v} v
$$

and hence it commutes with the $q$-Minkowski coordinates. It has the correct classical limit $\ell_{q=1}=$ $x_{0}^{2}-\vec{x}^{2}$.

We know from (4.3) that for $q$ phase $(|q|=1)$ the commutation relations (6.5) are preserved by an anti-linear anti-involution $\omega$ acting as :

$$
\omega\left(x_{ \pm}\right)=x_{ \pm}, \quad \omega(v)=\bar{v}
$$

from which follows also that $\omega\left(\ell_{q}\right)=\ell_{q}$.

The commutation rules of the spin variables $\bar{z}, z$ between themselves, with the $q$-Minkowski coordinates and with the $q$-Minkowski length are (cf. (4.2)) :

$$
\begin{aligned}
& x_{+} z=q^{-1} z x_{+}, \quad x_{-} z=q z x_{-}-\lambda v, \quad \bar{z} x_{+}=q x_{+} \bar{z}, \quad \bar{z} x_{-}=q^{-1} x_{-} \bar{z}+\lambda \bar{v} \\
& v z=q^{-1} z v, \quad \bar{v} z=q z \bar{v}-\lambda x_{+}, \quad \bar{z} v=q^{-1} v \bar{z}+\lambda x_{+}, \quad \bar{z} \bar{v}=q \bar{v} \bar{z} \\
& \bar{z} z=z \bar{z}, \quad z \ell_{q}=\ell_{q} z, \quad \bar{z} \ell_{q}=\ell_{q} \bar{z}
\end{aligned}
$$

Certainly, the commutation relations (6.23) are also preserved (for $q$ phase) by the conjugation $\omega$ which acts (cf. (4.3)) by : $\omega(z)=\bar{z}$. Thus, with this conjugation $\mathscr{Y}_{q}$ becomes a flag manifold of $S U_{q}(2,2)$. 
We know the normally ordered basis of the $q$ - deformed flag manifold $\mathscr{Y}_{q}$ considered as an associative algebra :

$$
\hat{\varphi}_{i j k \ell m n}=z^{i} v^{j} x_{-}^{k} x_{+}^{\ell} \bar{v}^{m} \bar{z}^{n}, \quad i, j, k, \ell, m, n \in \mathbb{Z}_{+}
$$

Let us denote by $\mathscr{Z}, \overline{\mathcal{Z}}$, and $\mathscr{M}_{q}$ the associative algebras with unity generated by $z, \bar{z}$, and $x_{ \pm}, v, \bar{v}$, resp. These three algebras are subalgebras of $\mathscr{Y}_{q}$, and we notice the following structure of $\mathscr{Y}_{q}:$

$$
\mathscr{Y}_{q} \cong \mathscr{Z} \mathscr{M}_{q} 刃 \overline{\mathscr{Z}}
$$

where $A \Subset B$ denotes the tensor product of $A$ and $B$ with $A$ acting on $B$.

We introduce now the representation spaces $C^{\chi}, \chi=\left[n_{1}, n_{2} ; d\right]$. The elements of $C^{\chi}$, which we shall call (abusing the notion) functions, are polynomials in $z, \bar{z}$ of degrees $n_{1}, n_{2}$, resp., and formal power series in the $q$ - Minkowski variables. (In the general $U_{q}(s l(n)$ ) situation the signatures $n_{1}, n_{2}$ are complex numbers and the functions are formal power series in $z, \bar{z}$ too, cf. $(B .2 \mathrm{~b}$ b).) Namely, these functions are given by:

$$
\hat{\varphi}_{n_{1}, n_{2}}(\bar{Y})=\sum_{\substack{i, j, k, \ell, m, n \in \mathbb{Z}_{+} \\ i \leq n_{1}, n \leq n_{2}}} \mu_{i j k \ell m n}^{n_{1}, n_{2}} \hat{\varphi}_{i j k \ell m n}
$$

where $\bar{Y}$ denotes the set of the six coordinates on $\mathscr{Y}_{q}$. Thus the analogs of $F_{v}^{ \pm}, J_{v}$, cf. (6.13), are :

$$
{ }_{q} F_{v}^{+}=\hat{\varphi}_{v+1, v-1}(\bar{Y}), \quad{ }_{q} F_{v}^{-}=\hat{\varphi}_{v-1, v+1}(\bar{Y}), \quad{ }_{q} J_{v}=\hat{\varphi}_{v, v}(\bar{Y})
$$

Next, analogously to the operators $\hat{M}_{j \ell}, T_{j \ell}, \hat{\mathscr{D}}_{j \ell}$, from (B.22), (??), (B.23]), we introduce operators $\hat{M}_{\kappa}, T_{\kappa}, \hat{\mathscr{D}}_{\kappa}$. We use these to write down explicitly the operators ${ }_{q} I_{V}^{ \pm}$in (G.J7). We have:

$$
\begin{aligned}
{ }_{q} I_{v}^{+}= & \frac{q^{2}}{2}[v+1]_{q}\left(q \hat{\mathscr{D}}_{v} T_{-}+\hat{M}_{\bar{z}} \hat{\mathscr{D}}_{+}\left(T_{v} T_{+}\right)^{-1} T_{\bar{v}}\right) T_{z}^{2}\left(T_{v} T_{\bar{z}}\right)^{-1}- \\
& -\frac{1}{2} q^{-v-1}\left(q \hat{M}_{z} \hat{\mathscr{D}}_{v} T_{-}+\hat{\mathscr{D}}_{-}+\hat{M}_{z} \hat{M}_{\bar{z}} \hat{\mathscr{D}}_{+}\left(T_{v} T_{+}\right)^{-1} T_{\bar{v}}+q^{-1} \hat{M}_{\bar{z}} \hat{\mathscr{D}}_{\bar{v}}\left(T_{-} T_{+}\right)^{-1}-\right. \\
& \left.-\lambda \hat{M}_{v} \hat{M}_{\bar{z}} \hat{\mathscr{D}}_{-} \hat{\mathscr{D}}_{+}\left(T_{-} T_{+}\right)^{-1} T_{\bar{v}}\right) \hat{\mathscr{D}}_{z} T_{z}\left(T_{v} T_{\bar{z}}\right)^{-1} \\
{ }_{q} I_{v}^{-}= & \frac{q}{2}[v+1]_{q}\left(q^{-1} \hat{\mathscr{D}}_{\bar{v}}\left(T_{-} T_{+}\right)^{-1}+\hat{M}_{z} \hat{\mathscr{D}}_{+}\left(T_{v} T_{+}\right)^{-1} T_{\bar{v}}-\right. \\
& \left.-\lambda \hat{M}_{v} \hat{\mathscr{D}}_{-} \hat{\mathscr{D}}_{+}\left(T_{-} T_{+}\right)^{-1} T_{\bar{v}}\right) T_{-} T_{+} T_{\bar{v}} T_{\bar{z}}+\frac{1}{2} q^{v+2}\left(q \hat{M}_{z} \hat{\mathscr{D}}_{v} T_{-}+\hat{\mathscr{D}}_{-}+\right. \\
& +\hat{M}_{z} \hat{M}_{\bar{z}} \hat{\mathscr{D}}_{+}\left(T_{v} T_{+}\right)^{-1} T_{\bar{v}}+q^{-1} \hat{M}_{\bar{z}} \hat{\mathscr{D}}_{\bar{v}}\left(T_{-} T_{+}\right)^{-1}- \\
& \left.-\lambda \hat{M}_{v} \hat{M}_{\bar{z}} \hat{\mathscr{D}}_{-} \hat{\mathscr{D}}_{+}\left(T_{-} T_{+}\right)^{-1} T_{\bar{v}}\right) \hat{\mathscr{D}}_{\bar{z}} T_{-} T_{+} T_{\bar{v}}
\end{aligned}
$$

With this the final result for the $q$ - Maxwell hierarchy of equations is (cf. (6.27)) :

$$
{ }_{q} I_{v}^{+}{ }_{q} F_{v}^{+}={ }_{q} J_{v}, \quad{ }_{q} I_{v}^{-}{ }_{q} F_{v}^{-}={ }_{q} J_{v}
$$

The final Figure below shows the place of the Maxwell hierarchy. 


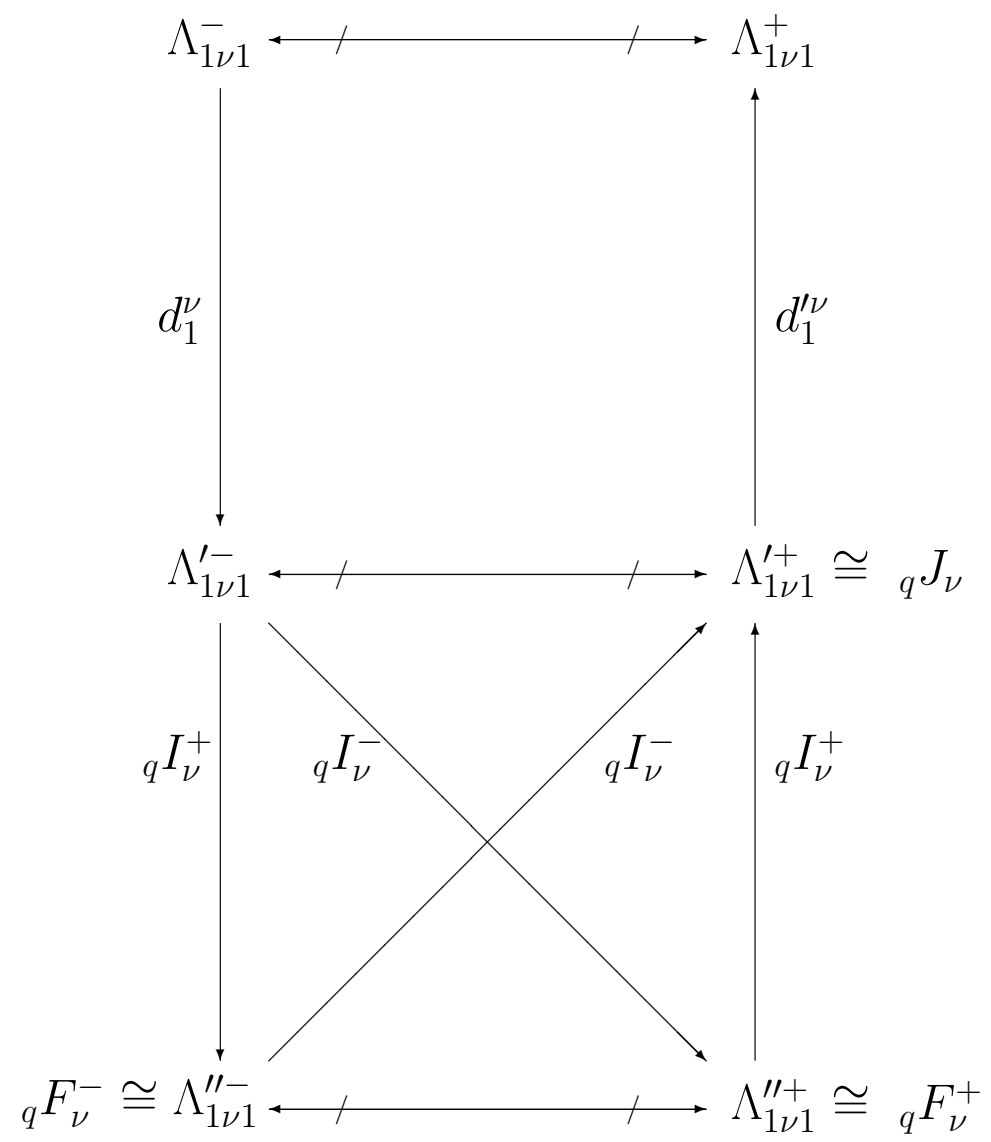

Fig. 4. The special case $p=n=1$ containing the Maxwell hierarchy 
Acknowledgments. The author would like to thank the Organizers for the kind invitation to speak at the Corfu Summer Institute 2015. The author has received partial support from the A.v. Humboldt Foundation and from COST action MP-1405.

\section{References}

[1] V.K. Dobrev, JHEP 1302 (2013) 015.

[2] V.K. Dobrev, Rep. Math. Phys. 25 (1988) 159-181.

[3] R.P. Langlands, "On the classification of irreducible representations of real algebraic groups", in: Representation Theory and Harmonic Analysis on Semi-Simple Lie Groups, eds. P. Sally, D.A. Vogan, Jr., Math. Surveys and Monographs, Vol. 31 (AMS, 1989) pp. 101-170.

[4] A.W. Knapp, G.J. Zuckerman, Ann. Math. 116 (1982) 389-501.

[5] I.N. Bernstein, I.M. Gel'fand, S.I. Gel'fand, Funkts. Anal. Prilozh. 5(1) 1-9(1971); English translation: Funct. Anal. Appl. 5 1-8 (1971).

[6] J. Dixmier, Enveloping Algebras, (North Holland, New York, 1977).

[7] Yu.I. Manin, Comm. Math. Phys. 123 (1989) 163-175.

[8] V.K. Dobrev, Representations of Quantum Groups and $q$ - Deformed Invariant Wave Equations, Dr. Habil. Thesis, Tech. Univ. Clausthal 1994, (Papierflieger Verlag, Clausthal-Zellerfeld, 1995) ISBN 3-930697-59-9.

[9] V.K. Dobrev, J. Phys. A: Math. Gen. 26 (1993) 1317-1334. 